\title{
COMPLETE CONTINUITY OF THE INVERSE OF A POSITIVE SYMMETRIC OPERATOR ${ }^{1}$
}

\author{
JAMES P. FINK
}

\begin{abstract}
Let $A$ be a symmetric positive definite linear transformation defined on a dense subset of a Hilbert space $H$, and let $H_{A}$ be the Hilbert space completion of the domain of $A$ with respect to the inner product $(u, v)_{A}=(A u, v)$. It is shown that the inverse of $A$ is completely continuous on $H_{A}$ if and only if it is completely continuous on $H$.
\end{abstract}

I. Introduction. In certain problems in mathematical physics, one of ten encounters differential operators which are defined on a dense subset of a Hilbert space and, under appropriate boundary conditions, are symmetric and semibounded. However, differential operators fail to possess certain desirable properties usually enjoyed by their inverses. One of these properties is complete continuity, and in this paper we show the equivalence of the complete continuity of this inverse in two Hilbert spaces (Theorem 2).

II. Preliminaries. Let $A$ be a symmetric linear transformation defined on a linear subset $M$ which is dense in a Hilbert space $H$. Suppose also that $A$ is positive definite on $M$; that is, suppose there exists a constant $\gamma>0$ such that

$$
(A u, u) \geqq \gamma^{2}\|u\|^{2}, \quad u \in M .
$$

Following the method due to K. Friedrichs [1] (cf. also [2], [3]), we define a new scalar product $(u, v)_{A}$ on $M$ by setting

$$
(u, v)_{A}=(A u, v), \quad u, v \in M .
$$

We denote the corresponding norm by

$$
\|u\|_{A}=(A u, u)^{1 / 2}, \quad u \in M .
$$

With this new metric, $M$ becomes an inner product space which can

Received by the editors April 28, 1969.

AMS Subject Classifications. Primary 4615, 4745; Secondary 69XX.

Key Words and Phrases. Linear transformations on Hilbert space, symmetric linear transformation, positive linear transformation, completely continuous linear transformation, inverse transformation, eigenvalues of completely continuous transformations, compact linear transformation.

1 Supported in part by the U. S. Atomic Energy Commission under Contract No. AT(30-1)-3829. 
be completed in the usual way, thereby obtaining a Hilbert space $H_{\mathbf{A}}$.

In the case we are considering, we have the following theorem:

THEOREM 1. The Hilbert space $H_{\mathbf{A}}$ can be identified with a subspace of $H$.

For a proof of this theorem, see [1] and also [2], [3].

It is easy to show that

$$
\begin{gathered}
(u, v)_{\boldsymbol{A}}=(A u, v), \quad u \in M, \quad v \in H_{\boldsymbol{A}}, \\
\|u\|_{\boldsymbol{A}} \geqq \gamma\|u\|, \quad u \in H_{\boldsymbol{A}} .
\end{gathered}
$$

Let $f$ be a fixed element in $H$ and consider the linear functional

$$
F_{f}(u)=(u, f), \quad u \in H_{\mathbf{A}} .
$$

$F_{f}$ is a bounded linear functional on $H_{A}$, and hence by the Riesz Representation Theorem there exists a unique element $u_{f} \in H_{\boldsymbol{A}}$ such that

$$
F_{f}(u)=(u, f)=\left(u, u_{f}\right)_{A}, \quad u \in H_{A} .
$$

We thus define an operator $G$ from $H$ into $H_{\boldsymbol{A}}$ by setting

$$
G f=u_{f}, \quad f \in H .
$$

We then have that

$$
(u, f)=(u, G f)_{A}, \quad u \in H_{A}, \quad f \in H .
$$

The properties of the operator $G$ are summarized in the following two propositions, the proofs of which can be extracted from [2] and [3].

Proposition 1. $G$ is a positive symmetric bounded linear transformation both on $H$ and on $H_{A}$.

Proposition 2. If $G f=0$ for $f \in H$, then $f=0$. In other words, $G$ has an inverse.

III. Complete continuity of $G$. In the applications, it is useful to know whether or not the operator $G$ is completely continuous (compact) as an operator on $H_{A}$. This question is not always as simple to answer as the related question of the complete continuity of $G$ as an operator on $H$. The next theorem provides the desired connection.

THEOREM 2. $G$ is completely continuous on $H_{\boldsymbol{A}}$ if and only if $G$ is completely continuous on $H$.

Proof. For the sake of clarity, we use the notation $\sum$ to denote 
summation in the $H$ metric of an infinite series and introduce the notation $\sum_{\boldsymbol{A}}$ to denote summation in the $H_{\boldsymbol{A}}$ metric.

Suppose first that $G$ is completely continuous on $H$. Since $G$ is also symmetric on $H$, its nonzero eigenvalues $\mu_{i}$, arranged according to decreasing absolute values, are of finite multiplicity and either finite or countably infinite in number (cf. [2]). In the latter case, $\mu_{i} \rightarrow 0$. Furthermore, every element of the form $G f, f \in H$, can be developed in terms of the orthonormal system $\left\{\phi_{i}\right\}$ of corresponding eigenvectors:

$$
G f=\sum\left(G f, \phi_{i}\right) \phi_{i}=\sum \mu_{i}\left(f, \phi_{i}\right) \phi_{i} .
$$

Since $G$ is a positive operator on $H, \mu_{i}>0$. By Proposition 2, 0 is not an eigenvalue of $G$ and consequently the eigenvectors $\phi_{i}$ corresponding to the nonzero eigenvalues $\mu_{i}$ form a complete orthonormal sequence in $H$ (cf. [2]). Since

$$
G \phi_{i}=\mu_{i} \phi_{i}, \quad \mu_{i}>0,
$$

we have that

$$
\phi_{i}=\mu_{i}^{-1} G \phi_{i} \in H_{A} .
$$

Setting $u_{i}=\mu_{i}^{1 / 2} \phi_{i}$, we observe that the $u_{i}$ form an orthonormal set in $H_{A}$.

Let $u \in H_{A}$ and set

$$
v=\sum_{A}\left(u, u_{i}\right)_{A} u_{i}
$$

Since $G$ is a continuous linear transformation on $H_{\boldsymbol{A}}$, we have that

$$
\begin{aligned}
G v & =\sum_{A}\left(u, u_{i}\right)_{A} G u_{i} \\
& =\sum_{A} \mu_{i}\left(u, u_{i}\right)_{A} u_{i} \\
& =\sum^{A} \mu_{i}\left(u, u_{i}\right)_{A} u_{i} .
\end{aligned}
$$

The last step follows because convergence in the $H_{\boldsymbol{A}}$ metric implies convergence in the $H$ metric.

Now $G$ is completely continuous and symmetric on $H$ so that

$$
\begin{aligned}
G u & =\sum \mu_{i}\left(u, \phi_{i}\right) \phi_{i} \\
& =\sum\left(u, u_{i}\right) u_{i} \\
& =\sum\left(u, G u_{i}\right)_{A} u_{i} \\
& =\sum \mu_{i}\left(u, u_{i}\right)_{A} u_{i} .
\end{aligned}
$$


Consequently, $G u=G v$ and $u=v$. Therefore, the $u_{i}$ form a complete orthonormal set in $H_{A}$ and

$$
G u=\sum_{A} \mu_{i}\left(u, u_{i}\right)_{A} u_{i}, \quad u \in H_{A} .
$$

It follows that $G$ is completely continuous on $H_{\boldsymbol{A}}$ (cf. [2]).

Conversely, suppose that $G$ is completely continuous on $H_{A}$. Since $G$ is symmetric on $H_{A}$, its nonzero eigenvalues $\mu_{i}$, arranged according to decreasing absolute values, are of finite multiplicity and either finite or countably infinite in number. In the latter case, $\mu_{i} \rightarrow 0$.

Since $G$ is a positive operator on $H_{A}, \mu_{i}>0$. By Proposition 2, 0 is not an eigenvalue of $G$ and consequently the eigenvectors $u_{i}$ corresponding to the nonzero eigenvalues $\mu_{i}$ form a complete orthonormal sequence in $H_{A}$. Setting $\phi_{i}=\mu_{i}^{-1 / 2} u_{i}$, we see that the $\phi_{i}$ form an orthonormal set in $H$.

Let $f \in H$. Then $G f \in H_{A}$ and

$$
G f=\sum_{\boldsymbol{A}}\left(G f, u_{i}\right)_{A} u_{i} .
$$

Since convergence in $H_{A}$ implies convergence in $H$, we obtain

$$
\begin{aligned}
G f & =\sum\left(G f, u_{i}\right)_{A} u_{i} \\
& =\sum\left(f, u_{i}\right) u_{i} \\
& =\sum \mu_{i}\left(f, \phi_{i}\right) \phi_{i} \quad f \in H .
\end{aligned}
$$

Hence, $G$ is completely continuous on $H$.

\section{REFERENCES}

1. K. Friedrichs, Spektraltheorie halbbeschränkter Operatoren und Anwendung auf die Spektralzerlegung von Differentialoperatoren, Math. Ann. 109 (1934), 465.

2. F. Riesz and B. Sz.-Nagy, Functional analysis, Akad. Kiad6, Budapest, 1953; English transl., Ungar, New York, 1955. MR 15, 132; MR 17, 175.

3. S. G. Mihlin, The problem of the minimum of a quadratic functional, GITTL, Moscow, 1952; English transl., Holden-Day Series in Mathematical Physics, HoldenDay, San Francisco, Calif., 1965. MR 16, 41; MR 30 \#1427.

University of Pittsburgh, Pittsburgh, Pennsylvania 15213 